\title{
Three-dimensional measurement and analysis of benign prostatic hyperplasia
}

\author{
Yankun Feng ${ }^{1 \#}$, Jianhui $\mathrm{Wu}^{2 \#}$, He Zhu ${ }^{3}$, Qiming Wang ${ }^{1}$, Tianyi $\mathrm{Li}^{1}$, Yong $\mathrm{Xu}^{4}$, Ping Zhang ${ }^{1}$, Lidong Zhai ${ }^{1}$ \\ ${ }^{1}$ Department of Anatomy and Histology, School of Basic Medical Sciences, Tianjin Medical University, Tianjin, China; ${ }^{2}$ Department of Urology, \\ Tianjin First Central Hospital, Tianjin, China; ${ }^{3}$ Department of Anesthesiology, Tianjin Central Hospital of Gynecology and Obstetrics, Tianjin, \\ China; ${ }^{4}$ Department of Urology, the Second Hospital of Tianjin Medical University, Tianjin Institute of Urology, Tianjin, China \\ Contributions: (I) Conception and design: L Zhai; (II) Administrative support: Y Xu, P Zhang; (III) Provision of study materials or patients: J Wu, \\ H Zhu; (IV) Collection and assembly of data: Y Feng, J Wu, T Li, Q Wang; (V) Data analysis and interpretation: Y Feng, J Wu, L Zhai; (VI) \\ Manuscript writing: All authors; (VII) Final approval of manuscript: All authors. \\ \#These authors contributed equally to this work. \\ Correspondence to: Lidong Zhai. Department of Anatomy and Histology, School of Basic Medical Sciences, Tianjin Medical University, 22 Qixiangtai \\ Road, Heping District, Tianjin 300070, China. Email: zhailidong2005@126.com.
}

Background: The volume and thickness of intravesical prostatic protrusion and other characteristics of benign prostatic hyperplasia have not been investigated. We determine the effects of age and prostate volume on anatomical features of benign prostatic hyperplasia using three-dimensional measurement in this study.

Methods: This retrospective study included a total of 98 patients with benign prostatic hyperplasia. Threedimensional models of prostate, central gland, peripheral zone, intravesical prostatic protrusion, prostatic urethra and bladder were reconstructed according to pelvic T2-weighted magnetic resonance imaging of these patients. The models were used to measure the intravesical prostatic protrusion volume, intravesical prostatic protrusion thickness, intravesical prostatic protrusion index, intravesical prostatic protrusion, prostate volume, peripheral zone volume, peripheral zone thickness, peripheral zone index, prostatic urethra thickness, the angle and distance of distal prostatic urethra with regard to coronal plane and sagittal plane and so on.

Results: Intravesical prostatic protrusion volume, intravesical prostatic protrusion thickness and peripheral zone volume of prostate volume $>80 \mathrm{~mL}$ group were significantly higher than these in prostate volume $<80 \mathrm{~mL}$ group $(\mathrm{P}<0.001,0.01,0.01$, respectively). These parameters significantly increased with age $(\mathrm{P}<0.001$, $0.01,0.05$, respectively). Peripheral zone index was significantly lower of prostate volume $>80 \mathrm{~mL}$ group than these in prostate volume $<80 \mathrm{~mL}$ group $(\mathrm{P}<0.05)$. Peripheral zone index significantly decreased with age $(\mathrm{P}<0.01)$. Intravesical prostatic protrusion index had no significant difference in all age groups. Peripheral zone thickness and prostatic urethra thickness had no significant difference in all groups. The distance and angle of distal prostatic urethra prostatic urethra with regard to coronal plane were significantly higher than these with regard to sagittal plane (both $\mathrm{P}<0.001$ ).

Conclusions: The rearward slope of the prostatic urethra is greater than the left or right offset during the process of benign prostatic hyperplasia. Three-dimensional measurement provides good supports for further clinical and scientific research.

Keywords: 3D measurement; benign prostatic hyperplasia (BPH); intravesical prostatic protrusion; peripheral zone; prostatic urethra

Submitted Feb 20, 2021. Accepted for publication Apr 22, 2021.

doi: $10.21037 /$ tau-21-142

View this article at: http://dx.doi.org/10.21037/tau-21-142 


\section{Introduction}

Benign prostatic hyperplasia (BPH) is associated with lower urinary tract symptoms and bladder outlet obstruction. Many non-invasive parameters related to benign prostatic hyperplasia have been widely measured in two-dimensional (2D) planes. The parameters are characterized by noninvasive and convenient comparison, and can be used for BPH diagnosis (1). With the advancement of research, the prostatic urethral has gained much attention. During the hyperplasia process of prostate, the length of prostatic urethra and prostatic urethra angle will be altered (2). Although these changes have been measured in 2D planes (3-5), the degree of which the prostatic urethra is compressed by the prostate and the angle relationship between prostate urethral and bladder have not been investigated. Prostate volume (PV) has attracted considerable attention in 2D planes (6). However, it is difficult to measure intravesical prostatic volume (IPPV), intravesical prostatic thickness (IPPT) and intravesical prostatic protrusion surface area (IPPS).

Three-dimensional (3D) reconstruction can be used to measure the PV $(7,8)$ and the changes of bladder wall thickness (BWT) (9). Its advantages are accurate and intuitive. However, other 2D parameters related to $\mathrm{BPH}$ have not been measured in $3 \mathrm{D}$ planes as well as other characteristics of prostate, prostate urethral, and bladder. In addition, little attention has been paid to the $3 \mathrm{D}$ reconstruction of prostate zonal anatomy.

We measured conventional parameters in $2 \mathrm{D}$ plane, as well as other features of BPH using three-dimensional measurement in this study. The purpose of this research is to better understanding the lower urinary tract anatomy of male and to provide references for clinical diagnosis and treatment of BPH. We present the following article in accordance with the STROBE reporting checklist (available at http://dx.doi.org/10.21037/tau-21-142).

\section{Methods}

\section{Study population}

This retrospective study analyzed clinical database of patients who visited Tianjin first central hospital for lower urinary tract symptoms/BPH from October 2016 to November 2019. A detailed history of 296 patients who underwent T2 MRI was obtained in our study. Exclusion criteria included: history of surgery of lower urinary tract, prostatitis and other non-benign prostatic hyperplasia diseases, patients did not carry a urinary catheter. Patients whose peripheral zone and/or central gland were impossible to identify were also excluded from this study. Finally, 98 patients who carried a urinary catheter with urinary retention which was caused by benign prostatic hyperplasia were eligible for analysis. They were divided into 4 groups by age: $50-60,60-70,70-80$ and $80-90\left(\mathrm{n}_{50-60}=14, \mathrm{n}_{60-70}=30\right.$, $\left.\mathrm{n}_{60-70}=36, \mathrm{n}_{70-80}=18\right)$. The study was conducted in accordance with the Declaration of Helsinki (as revised in 2013) and approved by Institutional Review Board (IRB) of the Tianjin First Center Hospital (registration ID2018NO22KY). Individual consent for this retrospective analysis was waived.

\section{$3 D$ reconstruction}

Patients underwent T2-weighted MRI using a 3-Tesla magnet coil with a slice thickness of $3 \mathrm{~mm}$. The first step, the collected MRI data from patients were imported into Mimics 19.0 software (Materialize, Leuven, Belgium) in DICOM file for 3D reconstruction of prostate, central gland, peripheral zone, intravesical prostatic protrusion, prostatic urethra and bladder based on horizontal, sagittal and coronal of pelvic T2-weighted MRI. To achieve accurate reconstruction of the lower urinary tract anatomy, this study applies corresponding 3D model-based registration for creation of fusion 3D model on account of each horizontal, sagittal and coronal plane. The Second step, the corresponding 3D models were fitted precisely in Geomagic Studio 14.0 software (Geomagic, Rock Hill, SC, USA) based on the same coordinate system. At the same time, the results of parameters related to $2 \mathrm{D}$ plane were measured. Finally, 3D measurement and analysis of related parameters were performed in 3-matic software (Materialize, Leuven, Belgium).

\section{Parameter measurements}

In $2 \mathrm{D}$ plane, the highest diameter of the prostate (HD) was measured on the maximum sagittal section. The longest diameter of the prostate (LD) and the widest diameter of the prostate (WD) were measured on the maximum horizontal section. Then, PV was calculated according to the ellipse volume formula: $0.52 \times \mathrm{LD} \times \mathrm{WD} \times \mathrm{HD}$. The measurement of IPP was completed on the sagittal section. A straight line from the left lowest point to the right lowest point where the prostate protrudes into the bladder was drawn. The distance from the highest point of the intravesical prostatic protrusion to this line was defined as IPP. 
In $3 \mathrm{D}$ plane, the following parameters were measured or analyzed by 3-matic software. IPPV, intravesical prostatic protrusion surface area (IPPS), IPPT, PV, prostate thickness (PT), prostate surface area (PS), central gland volume (CGV), central gland thickness (CGT), central gland surface area (CGS), peripheral zone volume (PZV), peripheral zone thickness (PZT), peripheral zone surface area (PZS), prostatic urethra thickness (PUT) and BWT were analyzed by 3 -matic software. Central gland index (CGI) was determined by dividing CGV by PV. Peripheral zone index $(\mathrm{PZI})$ was defined as the ratio $\mathrm{PZV} / \mathrm{PV}$. Intravesical prostatic protrusion index (IPPI) was defined as the ratio IPPV/PV. Based on the measurement of PV, they were divided into two groups: $<80 \mathrm{~mL}(\mathrm{n}=48)$ and $>80 \mathrm{~mL}(\mathrm{n}=50)$. LD, WD and HD were determined by 3-matic software from the highest and lowest points of the $\mathrm{X}$-axis, $\mathrm{Y}$-axis, and $\mathrm{Z}$-axis in the world coordinate system, respectively.

The straight line which was used to measure IPP on the 2D plane previously was imported into 3-matic software. The plane in which the intravesical prostatic protrusion lies was established by 3 -matic software was based on this straight line. The highest point of intravesical prostatic protrusion was taken as the endmost of intravesical prostatic protrusion tangent to a plane which is parallel to the previously established plane. The distance from the highest point to the plane was defined as IPP. The center of gravity of prostate was analyzed by 3 -matic software, and the coronal plane and sagittal plane were built by 3 -matic software based on the center of gravity of prostate. The central line of proximal prostatic urethra and distal prostatic urethra were analyzed by 3 -matic software. The angle between the two central lines was defined as prostatic urethra angle (PUA). The angle of central line of the proximal prostatic urethra with respect to the coronal plane and sagittal plane were defined as the $\mathrm{A}$-angle and $\mathrm{B}$-angle, respectively. The angle of the central line of distal prostatic urethra with respect to the coronal plane and sagittal plane were defined as the $\mathrm{C}$-angle and $\mathrm{D}$-angle, respectively. The angle between the bladder and prostatic urethra was defined as the E-angle. The highest point and the lowest point of prostatic urethra were analyzed by 3 -matic software. Then, the distance from the highest point to the coronal plane and sagittal plane were defined as the A-Distance and B-Distance, respectively. The distance from the lowest point to the coronal plane and sagittal plane were defined as the C-Distance and D-Distance, respectively. The surface distance from proximal endpoint of prostatic urethra to distal endpoint of prostatic urethra was defined as prostatic urethra length (PUL).

\section{Statistical analysis}

Values were shown as mean \pm standard deviation. Two independent-samples $t$-tests or Mann-Whitney $\mathrm{U}$ test were used to compare the parameters of $\mathrm{PV}<80 \mathrm{~mL}$ group and $\mathrm{PV}>80 \mathrm{~mL}$ group as well as $2-\mathrm{D}$ measurements and $3 \mathrm{D}$ measurements. All parameters of proximal prostatic urethra and distal prostatic urethra were compared by the paired $t$-test or Wilcoxon Rank-Sum test. Comparisons between age groups were made by one-way ANOVA or KruskallWallis test. All statistical methods were chosen according to whether the data conformed to normal distribution. All statistical analysis was analyzed by SPSS software 25.0 (IBM, Armonk, NY, USA). P<0.05 considered statistically significant.

\section{Results}

According to the T2-weighted MRI of patients, 3D models of prostate, intravesical prostatic protrusion, central gland, peripheral zone, bladder, and prostatic urethra were successfully reconstructed. These models can be observed from different points of view (Figure 1). Moreover, many parameters of the modes were able to be measured and analyzed by Mimics software, such as angle, diameter line, distance and thickness (Figures 2,3). Table 1 showed the mean measurements of all BPH parameters. Age, PV, IPPV, CGV, PZV, IPPI, CGI, LD, WD, HD, IPP, PUL, A-Distance, A-Angle, PT, IPPT, CGT, PS, IPPS, CGS, $\mathrm{PZS}$ of $\mathrm{PV}>80 \mathrm{~mL}$ group were significantly higher than these in $\mathrm{PV}<80 \mathrm{~mL}$ group $(\mathrm{P}<0.001,0.001,0.001,0.001$, $0.01,0.05,0.01,0.001,0.001,0.001,0.01,0.001,0.01,0.05$, $0.001,0.01,0.001,0.001,0.001,0.001,0.001$, respectively). $\mathrm{PZI}$ of $\mathrm{PV}<80 \mathrm{~mL}$ group was significantly higher than it in the $\mathrm{PV}>80 \mathrm{~mL}$ group $(\mathrm{P}<0.05)$. No significant difference was noted in B-Distance, C-Distance, D-Distance, PUA, B-Angle, C-Angle, D-Angle, E-Angle, PZT, BWT, PUT between the two groups (Table 2).

The WD was significantly higher in $3 \mathrm{D}$ measurement than it in $2 \mathrm{D}$ measurement (mean $57.79 \pm 8.85 \mathrm{vs}$. $50.42 \pm 9.06, \mathrm{P}<0.001)$. Meanwhile, LD $(\mathrm{P}<0.05)$. IPP, PV, $\mathrm{LD}$ and $\mathrm{HD}$ in $2 \mathrm{D}$ measurement and $3 \mathrm{D}$ measurement were not significantly different (Table 3). A-Distance and A-Angle were significant higher than B-Distance and $\mathrm{B}$-Angle, respectively (both $\mathrm{P}<0.001$ ). C-Distance 

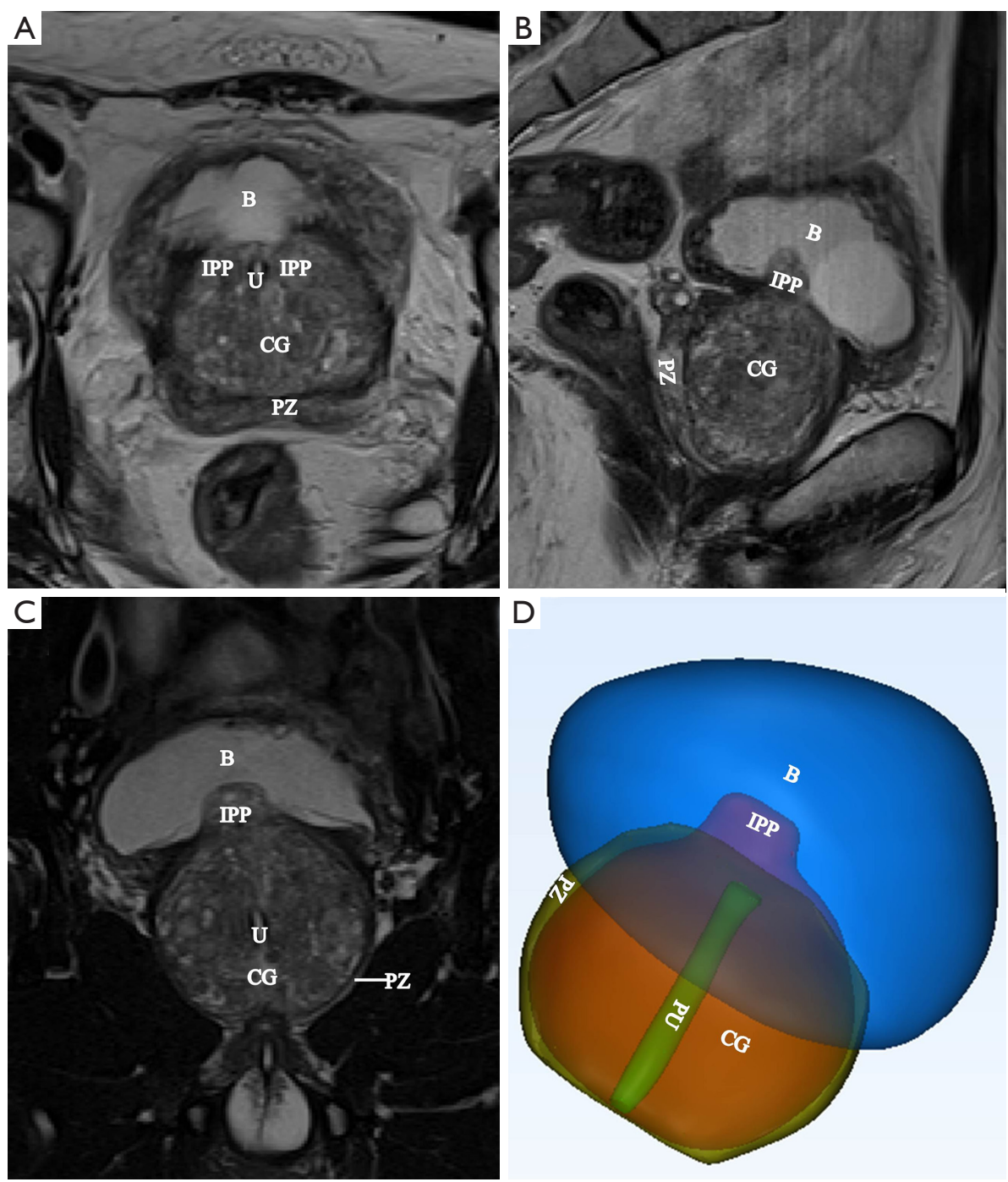

Figure $13 \mathrm{D}$ reconstruction of lower urinary tract based on T2-weighted MRI. (A) The horizontal scanning image. (B) The sagittal scanning image. (C) The coronal scanning image. (D) 3D reconstruction of bladder, prostatic urethra, central gland, peripheral zone and intravesical prostatic protrusion. 3D, three-dimensional; MRI, magnetic resonance imaging; B, bladder; CG, central gland; IPP, intravesical prostatic protrusion; PU, Prostatic urethra; PZ, peripheral zone; U, urethra.

and C-Angle were significantly higher than D-Distance and $\mathrm{D}$-Angle, respectively (both $\mathrm{P}<0.001$ ). A-Distance, B-Distance, A-Angle and B-Angle were significant higher than C-Distance, D-Distance, C-Angle and D-Angle, respectively (all $\mathrm{P}<0.001)$ (Table 4).

Moreover, PV, IPPV, CGV, PZV, CGI, LD, WD, HD, IPP, PUL, A-Distance, PT, IPPT, CGT, PS, IPPS, CGS and PZS remarkably increased with age $(\mathrm{P}<0.001,0.001$, $0.001,0.05,0.01,0.001,0.001,0.001,0.01,0.001,0.001$, 0.001, 0.01, 0.001, 0.001, 0.001, 0.001, 0.001, respectively), while PZI significantly decreased with age $(\mathrm{P}<0.01)$.
However, there were no significant differences in IPPI, B-Distance, C-Distance, D-Distance, PUA, A-Angle, B-Angle, C-Angle, D-Angle, E-Angle, PZT, BWT and PUT in different age categories (Table 5).

\section{Discussion}

MRI was a robust and reliable method for measuring PV (10). MRI with 3D segmentation software could provide an effective solution for accurate measurements of PV (11). In this study, 3D measurement was used not only 
A

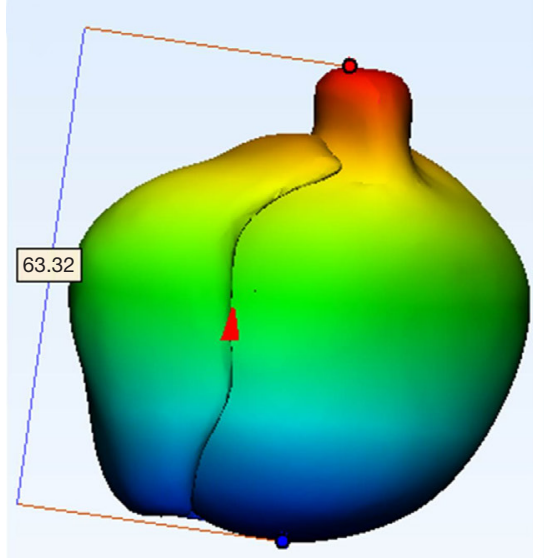

D

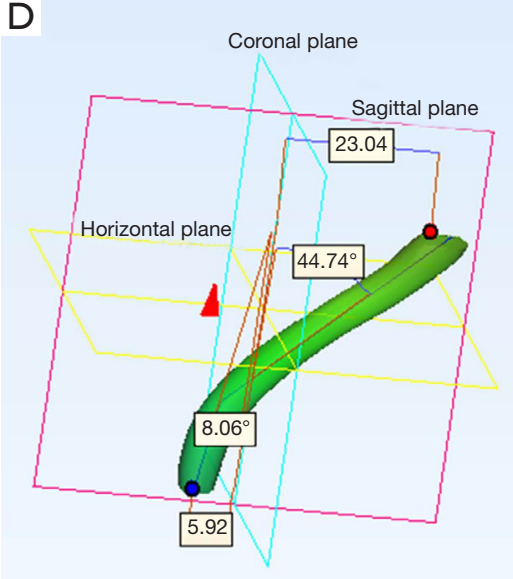

B

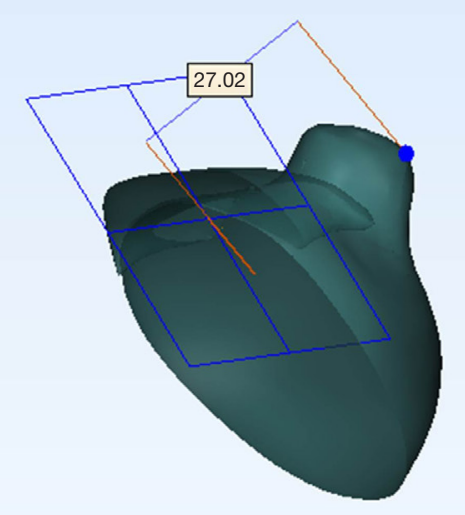

$\mathrm{E}$

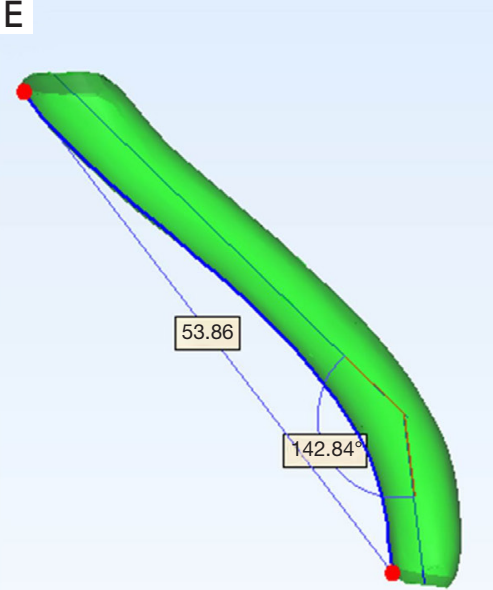

C

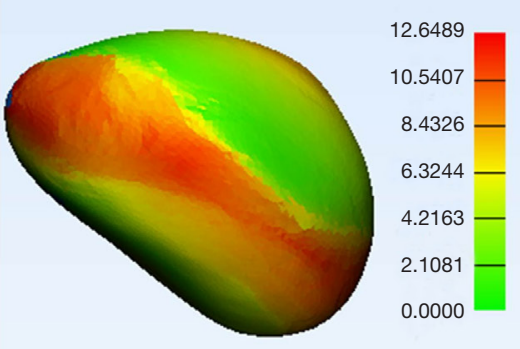

$\mathrm{F}$

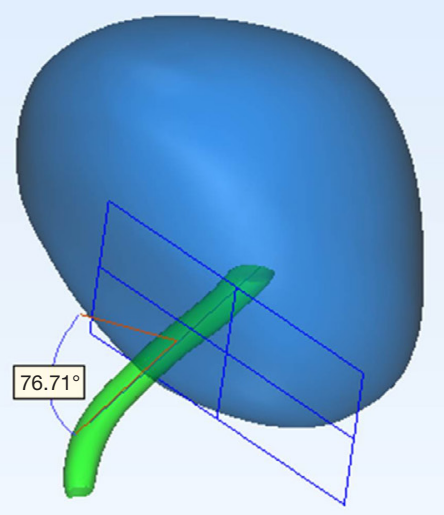

Figure $23 \mathrm{D}$ analysis of length $(\mathrm{mm})$, thickness $(\mathrm{mm})$ and angle $\left({ }^{\circ}\right)$. (A) The highest and lowest point of prostate were analyzed by 3 -matic software, the HD was measured from the highest point to the lowest point. The HD is $63.32 \mathrm{~mm}$. (B) The plane of IPP was fitted by 3 -matic software according to the straight line which was used to measure IPP on 2D plane. The highest point of IPP was taken as the endmost of IPP tangent to a plane which is parallel to the plane of IPP. The IPP is $27.02 \mathrm{~mm}$. (C) BWT was analyzed by 3-matic software. The biggest BWT is $12.6489 \mathrm{~mm}$. (D) The central line of proximal prostatic urethra and distal prostatic urethra, the highest and lowest point of prostatic urethra were determined by 3-matic software, respectively. The angle of central line of the proximal prostatic urethra and distal prostatic urethra with respect to the coronal plane was defined as the $\mathrm{A}$-angle and $\mathrm{C}$-angle, respectively. The distance from the highest and lowest point of prostatic urethra to the coronal plane was defined as the A-Distance and C-Distance, respectively. The A-angle, C-angle, A-Distance and C-Distance were $44.74^{\circ}, 8.06^{\circ}, 23.04 \mathrm{~mm}$ and $5.92 \mathrm{~mm}$ respectively. (E) The angle between the two central lines was defined as PUA. The PUA is $142.84^{\circ}$. Surface distance from proximal endpoint of prostatic urethra to distal endpoint of prostatic urethra was defined as PUL. The PUL is $53.86 \mathrm{~mm}$. (F) The plane of bladder was fitted by 3-matic software based on the basilar part of bladder neck. The angle between the plane of bladder and the central line of proximal prostatic urethra was E-Angle. The E-Angle is $76.71^{\circ}$. HD, the highest diameter of prostate; IPP, intravesical prostatic protrusion; BWT, bladder wall thickness; PUA, Prostatic urethra angle; PUL, Prostatic urethra length.

to measure various $2 \mathrm{D}$ parameters related to $\mathrm{BPH}$ but also other characteristics of BPH. This study showed that $3 \mathrm{D}$ measurement can be used as a mean to understand the characteristics and pathology progression in $\mathrm{BPH}$.
This study found that 3D plane can provide us with much more accurate measurements than general 2D plane. There was statistical difference in the WD between the 2D measurement and 3D measurement. The WD had 

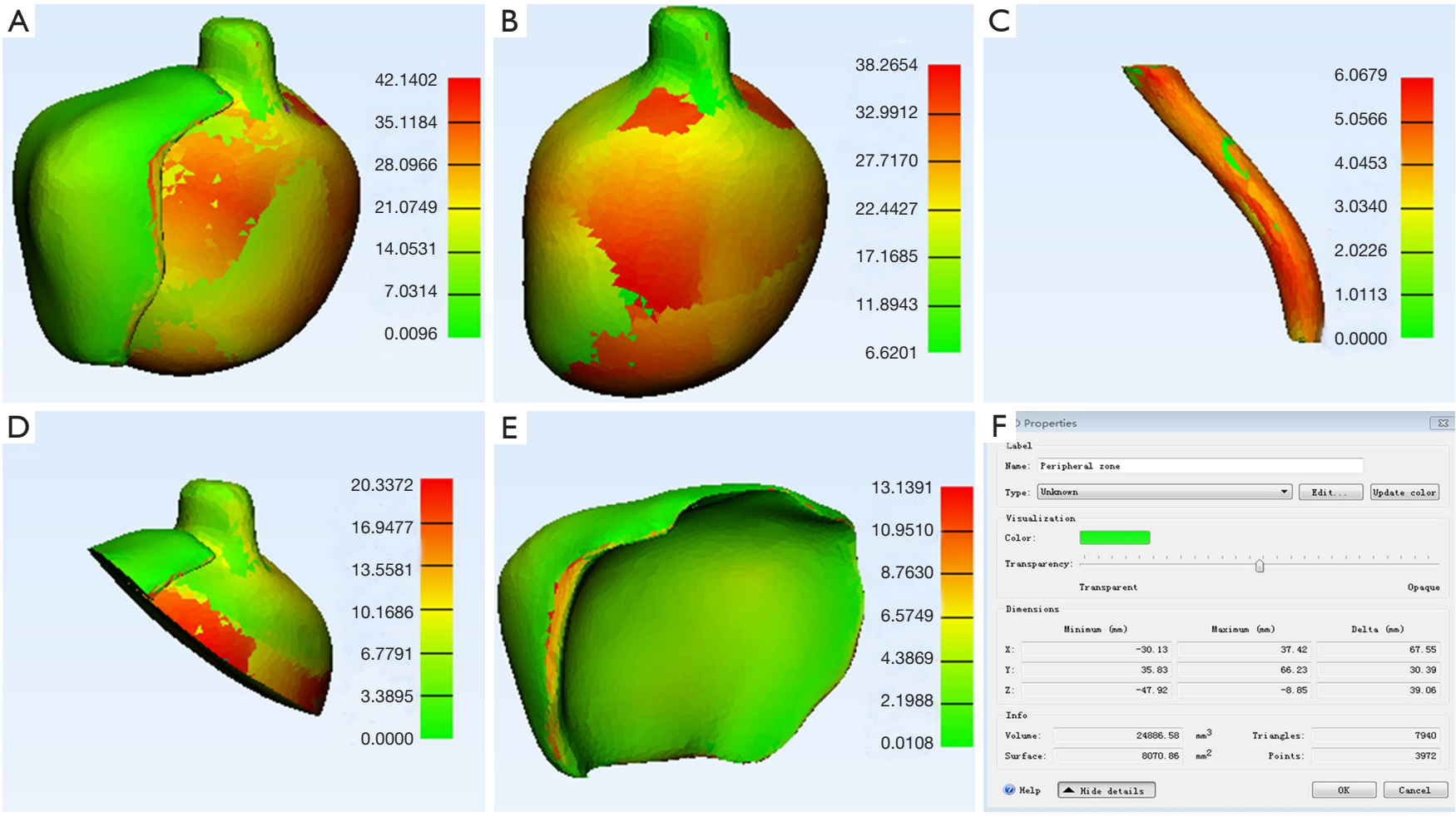

Figure 3 3D analysis of thickness $(\mathrm{mm})$, volume $\left(\mathrm{mm}^{3}\right)$ and surface area $\left(\mathrm{mm}^{2}\right)$. (A) Schematic diagram of analysis of PT. The biggest PT is $42.1402 \mathrm{~mm}$. (B) Schematic diagram of analysis of CGT. The biggest CGT is $38.2654 \mathrm{~mm}$. (C) Schematic diagram of analysis of PUT. The biggest PUT is $6.0679 \mathrm{~mm}$. (D) Schematic diagram of analysis of IPPT. The biggest IPPT is $20.3372 \mathrm{~mm}$. (E) Schematic diagram of analysis of PZT. The biggest PZT is $13.1391 \mathrm{~mm}$. (F) Schematic diagram of property of peripheral zone. The PZV is $24,886.58 \mathrm{~mm}^{3}$. The PZS is $8,070.86 \mathrm{~mm}^{2}$. PT, prostate thickness; CGT, central gland thickness; PUT, prostatic urethra thickness; IPPT, intravesical prostatic protrusion thickness; PZT, peripheral zone thickness; PZV, peripheral zone volume; PZS, peripheral zone surface area.

important implications in prostatectomy. Based on WD measurement, the anatomic structure of ejaculatory ducts can be determined, which had important implications for retention of ejaculatory function following transurethral resection of prostate (12). Measurement of diameter line in $2 \mathrm{D}$ planes is restricted by irregularities in prostate contour and the result is subjectively influenced by the operator. There may be a lack of anatomical features in the threedimensional model reconstructed by using the horizontal plane alone. To compensate for this defect and achieve accurate reconstruction of the lower urinary tract anatomy, this study applies 3D model-based registration for creation of fusion 3D model on account of each horizontal, sagittal and coronal plane. Moreover, measurement of the diameter line was analyzed by 3 -matic software. Therefore, $3 \mathrm{D}$ measurement guarantees more reliable and robust results.

Though formula of PV had been commonly explored, measurement errors were inevitable $(13,14)$. Measurement of PV was successfully achieved in this study. Measurement of the PV can serve as a reference standard to use of alpha blockers (15), perform prostatectomy (16), and select prostate biopsy site (17). The standard critical value of $\mathrm{PV}=80 \mathrm{~mL}$ was chosen for the prostatectomy (18). The IPP was the important parameter to assess BPH (19). IPP, IPPT, IPPV, IPPI and IPPS of PV $>80 \mathrm{~mL}$ group were significantly higher than those in $\mathrm{PV}<80 \mathrm{~mL}$ group in our study. IPP, IPPT and IPPS significantly increased with age. IPPV and PV also strikingly increased with age, while IPPI did not change with age. IPPI was defined as a ratio between IPPV and PV. This indicated that increment of volume was almost the same in the PV and IPPV.

Tian et al. and Kwon et al. had used transrectal ultrasound (TRUS) to measure the PZT. Tian et al. discovered that the mean value of PZT was $13.3 \pm 6.3 \mathrm{~mm}$, which was not modulated by age (20). However, Kwon et al. found that the mean value of PZT was $11.10 \pm 2.50 \mathrm{~mm}$, 
Table 1 Characteristics of patients with benign prostate hyperplasia

\begin{tabular}{lc}
\hline Variable & Mean \pm SD \\
\hline Age (years) & $70.67 \pm 9.32$ \\
PV $(\mathrm{mL})$ & $93.07 \pm 43.20$ \\
IPPV $(\mathrm{mL})$ & $15.54 \pm 13.73$ \\
CGV $(\mathrm{mL})$ & $72.74 \pm 42.69$ \\
PZV $(\mathrm{mL})$ & $18.71 \pm 10.37$ \\
IPPI & $0.15 \pm 0.10$ \\
CGI & $0.75 \pm 0.15$ \\
PZI & $0.23 \pm 0.13$ \\
LD $(\mathrm{mm})$ & $60.52 \pm 8.98$ \\
WD $(\mathrm{mm})$ & $57.79 \pm 8.85$ \\
HD $(\mathrm{mm})$ & $58.18 \pm 13.01$ \\
IPP $(\mathrm{mm})$ & $16.67 \pm 9.52$ \\
PUL $(\mathrm{mm})$ & $53.89 \pm 11.71$ \\
A-Distance $(\mathrm{mm})$ & $15.21 \pm 7.23$ \\
B-Distance $(\mathrm{mm})$ & $6.32 \pm 5.35$ \\
C-Distance $(\mathrm{mm})$ & $4.91 \pm 3.39$ \\
D-Distance $(\mathrm{mm})$ & $1.98 \pm 1.22$ \\
PUA $\left(^{\circ}\right)$ & $136.60 \pm 12.37$ \\
A-Angle $\left(^{\circ}\right)$ & $42.60 \pm 11.34$ \\
B-Angle $\left(^{\circ}\right)$ & $6.61 \pm 5.79$ \\
C-Angle $\left({ }^{\circ}\right)$ & $10.49 \pm 5.71$ \\
\hline & \\
\hline &
\end{tabular}

Table 1 (continued)

which was significantly decreased with age (21). Those results were different from the result of our present study. The mean value of PZT in this study was $13.75 \pm 3.77 \mathrm{~mm}$. The differences may result from the inaccuracy of results measured by TRUS. In TRUS, PZT measurement was required to determine the longest line which was draw from the center of the transitional zone to the outer margin of the peripheral zone. Apart from that, peripheral zone was relatively difficult to be differentiated in TRUS. The profile of the peripheral zone was clearer in T2-weighted MRI. Accordingly, peripheral zone was reconstructed precisely. Moreover, PZT was analyzed by 3 -matic software. The analysis tools employed to measure $\mathrm{PZT}$ was reliable, which guaranteed the quality of this data. PZT had no significant difference in the patients above the age of 50. Our result was consistent with Tian et al.
Table 1 (continued)

\begin{tabular}{lc}
\hline Variable & Mean \pm SD \\
\hline D-Angle $\left(^{\circ}\right)$ & $3.50 \pm 2.95$ \\
E-Angle $\left(^{\circ}\right)$ & $96.54 \pm 15.47$ \\
PT $(\mathrm{mm})$ & $37.65 \pm 5.66$ \\
IPPT $(\mathrm{mm})$ & $18.95 \pm 6.71$ \\
CGT $(\mathrm{mm})$ & $31.62 \pm 6.63$ \\
PZT $(\mathrm{mm})$ & $13.65 \pm 3.68$ \\
BWT $(\mathrm{mm})$ & $11.33 \pm 3.50$ \\
PUT $(\mathrm{mm})$ & $13.59 \pm 7.80$ \\
PS $\left(\mathrm{cm}^{2}\right)$ & $110.32 \pm 35.92$ \\
IPPS $\left(\mathrm{cm}^{2}\right)$ & $40.28 \pm 21.24$ \\
CGS $\left(\mathrm{cm}^{2}\right)$ & $99.41 \pm 37.17$ \\
PZS $\left(\mathrm{cm}^{2}\right)$ & $64.65 \pm 19.27$ \\
\hline
\end{tabular}

SD, standard deviation; PV, prostate volume; IPPV, intravesical prostatic protrusion volume; CGV, central gland volume; PZV, peripheral zone volume; IPPI, intravesical prostatic protrusion index; CGI, central gland index; PZI, peripheral zone index; LD, the longest diameter of prostate; WD, the widest diameter of prostate; HD, the highest diameter of prostate; IPP, intravesical prostatic protrusion; PUL, prostatic urethra length; PUA, prostatic urethra angle; PT, prostate thickness; IPPT, intravesical prostatic protrusion thickness; CGT, central gland thickness; PZT, peripheral zone thickness; BWT, bladder wall thickness; PUT, prostatic urethra thickness; PS, prostate surface area; IPPS, intravesical prostatic protrusion surface area; CGS, central gland surface area; PZS, peripheral zone surface area.

The shape of peripheral zone is irregular. It was too difficult to measure the PZV and to find a solution to the measurement of PZV. However, 3D measurement was a good way to solve this problem. Currently, a few researches about PZV were controversial. Matsugasumi et al. reported findings from a retrospective analysis that the PZV maintained steadily at about $20 \mathrm{~mL}$ in patients with $\mathrm{BPH}$, and the indirect influence of the age on the variation in PZV was small (22). However, findings from a longitudinal study by Williams et al. found that the PZV in the 50-60 years age group was significantly higher than it in $<45$ years age group, which increased about $5 \mathrm{~mL}$ (23). These differences were probably due to variations in study methodology.

Similar to study by Williams et al., we found that with the increased in PV, PZV was increasing as well, but the 
Table 2 Comparison of variables stratified by prostate volume

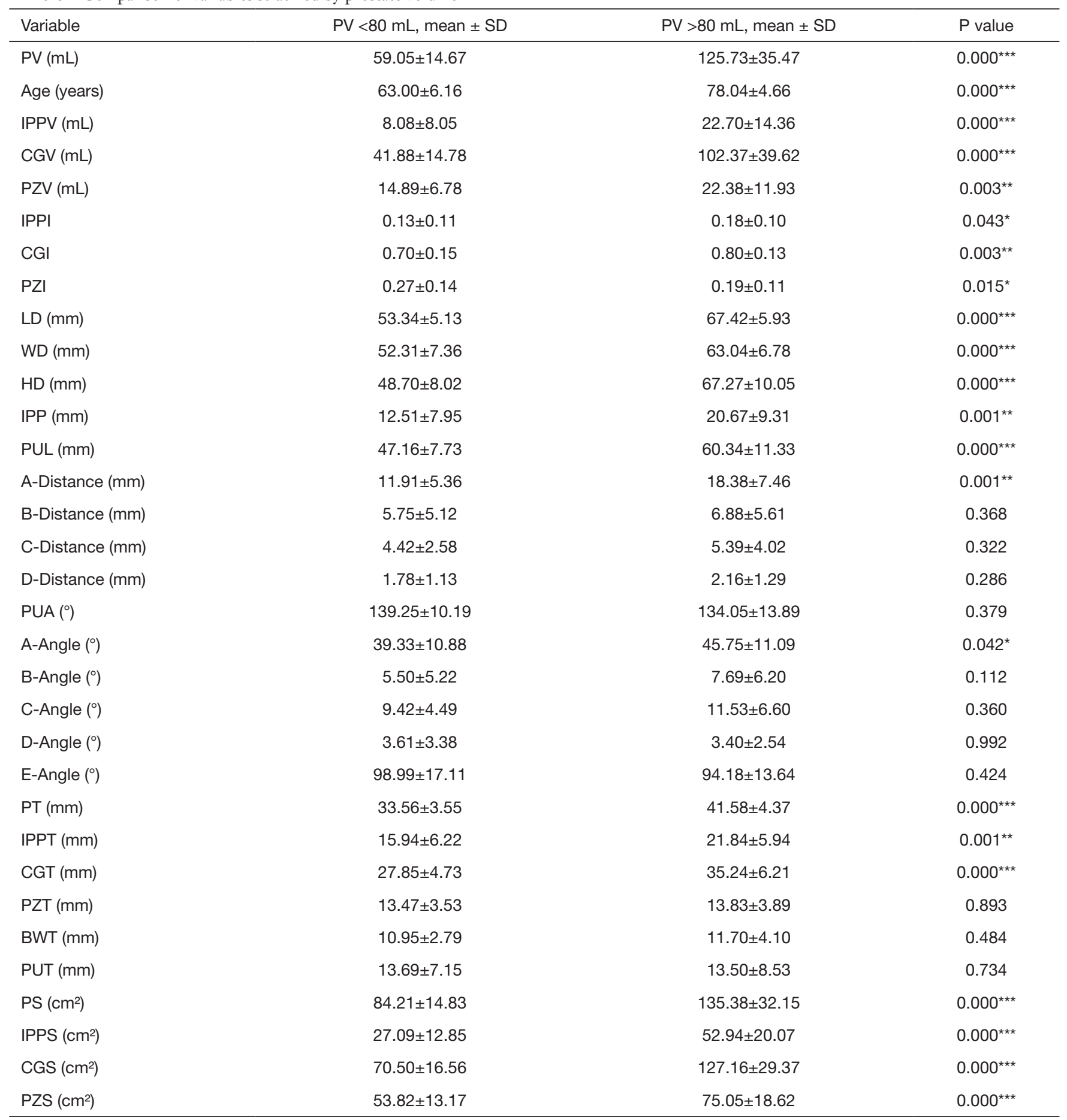

${ }^{\star} \mathrm{P}<0.05,{ }^{\star \star} \mathrm{P}<0.01,{ }^{* \star \star} \mathrm{P}<0.001$. SD, standard deviation; PV, prostate volume; IPPV, intravesical prostatic protrusion volume; CGV, central gland volume; PZV, peripheral zone volume; IPPI, intravesical prostatic protrusion index; CGI, central gland index; PZI, peripheral zone index; LD, the longest diameter of prostate; WD, the widest diameter of prostate; HD, the highest diameter of prostate; IPP, intravesical prostatic protrusion; PUL, prostatic urethra length; PUA, prostatic urethra angle; PT, prostate thickness; IPPT, intravesical prostatic protrusion thickness; CGT, central gland thickness; PZT, peripheral zone thickness; BWT, bladder wall thickness; PUT, prostatic urethra thickness; PS, prostate surface area; IPPS, intravesical prostatic protrusion surface area; CGS, central gland surface area; PZS, peripheral zone surface area. 
Table 3 Comparison between 2D measurements and 3D measurements

\begin{tabular}{lccc}
\hline Variable & 2D measurement, mean \pm SD & 3D measurement, mean \pm SD & P value \\
\hline LD $(\mathrm{mm})$ & $57.18 \pm 7.07$ & $60.52 \pm 8.98$ & $0.043^{\star}$ \\
WD $(\mathrm{mm})$ & $50.42 \pm 9.06$ & $57.79 \pm 8.85$ & $0.000^{\star \star \star}$ \\
HD $(\mathrm{mm})$ & $58.37 \pm 13.03$ & $58.18 \pm 13.01$ & 0.940 \\
IPP $(\mathrm{mm})$ & $17.91 \pm 9.60$ & $16.67 \pm 9.52$ & 0.390 \\
PV $(\mathrm{mL})$ & $93.18 \pm 43.70$ & $93.07 \pm 43.20$ & 0.990 \\
\hline
\end{tabular}

${ }^{*} \mathrm{P}<0.05,{ }^{* *} \mathrm{P}<0.001$. SD, standard deviation; $\mathrm{LD}$, the longest diameter of prostate; WD, the widest diameter of prostate; HD, the highest diameter of prostate; IPP, intravesical prostatic protrusion; PV, prostate volume.

Table 4 Comparison between the parameters of prostatic urethra (mean \pm SD)

\begin{tabular}{|c|c|c|}
\hline Variable & & $P$ value \\
\hline A-Angle $42.60 \pm 11.34$ & B-Angle $6.61 \pm 5.79$ & $0.000^{\star \star \star}$ \\
\hline C-Distance $4.91 \pm 3.39$ & D-Distance $1.98 \pm 1.22$ & $0.000^{\star \star \star}$ \\
\hline C-Angle $10.49 \pm 5.71$ & D-Angle $3.50 \pm 2.95$ & $0.000^{\star \star \star}$ \\
\hline A-Angle $42.60 \pm 11.34$ & C-Angle $10.49 \pm 5.71$ & $0.000^{\star \star *}$ \\
\hline B-Distance $6.32 \pm 5.35$ & D-Distance $1.98 \pm 1.22$ & $0.000^{\star \star *}$ \\
\hline B-Angle $6.61 \pm 5.79$ & D-Angle $3.50 \pm 2.95$ & $0.000^{\star \star \star}$ \\
\hline
\end{tabular}

${ }^{* \star *} \mathrm{P}<0.001 . \mathrm{SD}$, standard deviation.

magnitude of this change is relatively small. Comparing to 50-59 years age group and 60-69 years age group, the PZV for 60-69 years age group and 70-79 years age group increased few. In addition, this study found that PZI significantly decreased with age. This indicated that greater increases in the PV than in the PZV. Subjects in study by Matsugasumi et al. were Japanese and Americans. While in the Williams's study and our research, the subjects were Americans and Chinese respectively. Therefore, the discrepancy might be caused by the different races.

Comparing to $\mathrm{PV}<80 \mathrm{~mL}$ group, the $\mathrm{PZV}$ of $\mathrm{PV}>80 \mathrm{~mL}$ group increased also few. However, the PZI of PV $<80 \mathrm{~mL}$ group was significantly higher than the PV $>80 \mathrm{~mL}$ group. PZT showed no significant difference between PV $<80 \mathrm{~mL}$ group and $\mathrm{PV}>80 \mathrm{~mL}$ group. CGT and PT sharply increased with $\mathrm{PV}$ as well as age. This could be the reason why the correlation between $\mathrm{BPH}$ and transition zone was significantly higher than peripheral zone (24). PS, CGV, CGS, PZS, LD, WD and HD significantly increased with age as well as PV in our study. During the process of
$\mathrm{BPH}$, the prostate got closer to a circle. These results were consistent with the PCAR theory (25).

In 2D planes, PUA had a different definition. PUA was defined as the angle between line from seminal colliculus to bladder neck and rostocaudal axis (4). The vertex angle formed by two rays including both the proximal and distal prostatic urethra was also defined as PUA (26). In contrast to those, considering the characteristics of $3 \mathrm{D}$ plane, we used the same criteria to define PUA alteration as study by Ko et al. (2). The PV was increased, leading to prolongation of PUL. The PUL significantly increased with age. However, the PUA decreased with PV although the range was slight. The result was consistent with study by Chen et al. (27). A study by El-Tatawy et al. revealed that PUA did not show a significant difference in age (28), which was concordant with finding of our study. There were no significant differences in PUA between different age groups. With reference to coronal plane and sagittal plane, the angle and distance of which the proximal prostatic urethra was compressed by the prostate were higher than distal 
Table 5 Comparison of measurements in the four age groups (mean \pm SD)

\begin{tabular}{|c|c|c|c|c|c|}
\hline Variable & $50-60$ years & $60-70$ years & 70-80 years & 80-90 years & $P$ value \\
\hline IPPV (mL) & $4.44 \pm 3.40$ & $7.30 \pm 4.47$ & $19.60 \pm 13.00$ & $29.77 \pm 15.13$ & $0.000^{\star \star \star}$ \\
\hline CGV (mL) & $27.28 \pm 11.30$ & $45.45 \pm 9.86$ & $78.12 \pm 19.86$ & $142.85 \pm 32.15$ & $0.000^{\star \star \star}$ \\
\hline PZV (mL) & $12.06 \pm 5.95$ & $16.65 \pm 7.10$ & $21.46 \pm 12.88$ & $21.82 \pm 10.17$ & $0.040^{*}$ \\
\hline CGI & $0.65 \pm 0.20$ & $0.70 \pm 0.12$ & $0.77 \pm 0.14$ & $0.86 \pm 0.08$ & $0.002^{* *}$ \\
\hline PZI & $0.32 \pm 0.19$ & $0.26 \pm 0.11$ & $0.21 \pm 0.12$ & $0.14 \pm 0.07$ & $0.009^{\star \star}$ \\
\hline LD (mm) & $48.28 \pm 5.44$ & $55.52 \pm 3.52$ & $63.81 \pm 6.31$ & $71.82 \pm 3.14$ & $0.000^{\star \star \star}$ \\
\hline WD (mm) & $45.57 \pm 6.59$ & $54.88 \pm 5.71$ & $59.59 \pm 5.51$ & $68.54 \pm 5.65$ & $0.000^{\star * \star}$ \\
\hline A-Distance $(\mathrm{mm})$ & $8.49 \pm 4.70$ & $12.49 \pm 4.73$ & $16.62 \pm 6.17$ & $22.17 \pm 8.04$ & $0.000^{\star \star \star}$ \\
\hline B-Distance (mm) & $5.14 \pm 4.91$ & $4.70 \pm 3.22$ & $7.82 \pm 6.84$ & $6.95 \pm 4.90$ & 0.407 \\
\hline C-Distance (mm) & $3.85 \pm 2.88$ & $4.78 \pm 2.45$ & $4.69 \pm 2.96$ & $6.40 \pm 5.49$ & 0.480 \\
\hline D-Distance (mm) & $2.46 \pm 1.50$ & $1.52 \pm 0.88$ & $2.23 \pm 1.38$ & $1.85 \pm 1.02$ & 0.260 \\
\hline PUA $\left({ }^{\circ}\right)$ & $143.26 \pm 7.67$ & $137.74 \pm 11.53$ & $137.97 \pm 8.07$ & $126.77 \pm 18.69$ & 0.195 \\
\hline A-Angle $\left({ }^{\circ}\right)$ & $36.29 \pm 13.74$ & $39.56 \pm 9.88$ & $44.54 \pm 11.50$ & $48.71 \pm 8.73$ & 0.106 \\
\hline B-Angle $\left(^{\circ}\right)$ & $4.05 \pm 4.91$ & $4.89 \pm 3.69$ & $7.38 \pm 5.58$ & $9.95 \pm 8.23$ & 0.116 \\
\hline CGT (mm) & $24.57 \pm 4.05$ & $28.71 \pm 4.46$ & $32.96 \pm 4.57$ & $39.26 \pm 6.56$ & $0.000^{\star \star \star}$ \\
\hline PZT (mm) & $13.95 \pm 2.99$ & $13.66 \pm 3.86$ & $13.78 \pm 4.47$ & $13.16 \pm 2.40$ & 0.974 \\
\hline BWT (mm) & $10.59 \pm 3.48$ & $10.89 \pm 2.47$ & $11.06 \pm 3.06$ & $13.17 \pm 5.39$ & 0.382 \\
\hline PUT (mm) & $13.97 \pm 8.66$ & $13.75 \pm 7.06$ & $15.43 \pm 8.66$ & $9.35 \pm 5.89$ & 0.186 \\
\hline $\mathrm{PS}\left(\mathrm{cm}^{2}\right)$ & $65.72 \pm 9.86$ & $90.20 \pm 7.27$ & $115.99 \pm 21.51$ & $167.19 \pm 19.53$ & $0.000^{\star \star \star}$ \\
\hline IPPS (cm²) & $20.01 \pm 9.92$ & $26.79 \pm 9.05$ & $47.86 \pm 18.04$ & $63.38 \pm 19.16$ & $0.000^{\star \star \star}$ \\
\hline CGS $\left(\mathrm{cm}^{2}\right)$ & $54.08 \pm 16.64$ & $75.28 \pm 10.26$ & $107.47 \pm 13.45$ & $158.78 \pm 22.62$ & $0.000^{\star \star \star}$ \\
\hline $\mathrm{PZS}\left(\mathrm{cm}^{2}\right)$ & $45.14 \pm 12.55$ & $58.16 \pm 12.39$ & $69.89 \pm 16.57$ & $80.16 \pm 22.68$ & $0.000^{\star \star \star}$ \\
\hline
\end{tabular}

${ }^{*} \mathrm{P}<0.05$, ${ }^{* *} \mathrm{P}<0.01,{ }^{* \star *} \mathrm{P}<0.001$. SD, standard deviation; PV, prostate volume; IPPV, intravesical prostatic protrusion volume; CGV, central gland volume; PZV, peripheral zone volume; IPPI, intravesical prostatic protrusion index; CGI, central gland index; PZI, peripheral zone index; LD, the longest diameter of prostate; WD, the widest diameter of prostate; HD, the highest diameter of prostate; IPP, intravesical prostatic protrusion; PUL, prostatic urethra length; PUA, prostatic urethra angle; PT, prostate thickness; IPPT, intravesical prostatic protrusion thickness; CGT, central gland thickness; PZT, peripheral zone thickness; BWT, bladder wall thickness; PUT, prostatic urethra thickness; PS, prostate surface area; IPPS, intravesical prostatic protrusion surface area; CGS, central gland surface area; PZS, peripheral zone surface area. 
prostatic urethra in this study. Therefore, the degrees of the compression of proximal prostatic urethra were larger than distal prostatic urethra. With reference to coronal plane, the angle and distance of the proximal prostatic urethra and distal prostatic urethra were significantly higher than those with reference to sagittal plane. A-Distance and A-Angle of $\mathrm{PV}>80 \mathrm{~mL}$ group were significantly higher than those in $\mathrm{PV}$ $<80 \mathrm{~mL}$ group. A-Distance significantly increased with age.

This indicates that the rearward slope of the prostatic urethra is greater than the left or right offset during the process of BPH. To date, only one study has measured PUT. The PUT was measured as 6 [2-16] $\mathrm{mm}$ in a study by Guneyli et al. (29). However, the median (IQR) value of PUT was measured as $11.63(6.91-17.41) \mathrm{mm}$ in this study. The discrepancy might be due to patients had or had not a urinary catheter. Subjects in our study had a urinary catheter. The fine structure of prostatic urethra will be clearer and the reconstruction will be more accurate if patients had a urinary catheter. The PUT was analyzed by 3-matic software, which avoided the influence of subjective factors. No significant differences were found in PUT between any of the groups. This suggests BPH has little impacts on the PUT. No differences in BWT and E-Angle were also found between any of the groups. BWT was influenced by the volume of urine (30). Besides, patients who were unable to void may have some effect on BWT.

However, this study also has some limitations. First, while we were able to measure multiple features of $\mathrm{BPH}$, our choice of regions of interest was based on anatomical contour, which was time-consuming. Future work is needed to explore methods of automated $3 \mathrm{D}$ segmentation. In addition, the BWT and E-Angle results may be affected by the fact that the patients had a urinary catheter.

\section{Conclusions}

To sum up, 3D measurement is more accurate than $2 \mathrm{D}$ measurement. PZV was increasing, but the magnitude of this change is relatively small and the rearward slope of the prostatic urethra is greater than the left or right offset during the process of $\mathrm{BPH}$. The $\mathrm{BPH}$ has a minor impact on the PZT and PUT, and $3 \mathrm{D}$ measurement is a great tool for measuring features of $\mathrm{BPH}$, which provides good supports for further clinical and scientific research.

\section{Acknowledgments}

Funding: This work was supported by grants from the
National Natural Science Foundation of China (31871212, Lidong Zhai), Postdoctoral Science Foundation of China (2016M601272, Lidong Zhai), Chinese Clinical Trial Registry (ChiCTR1800014818, Jianhui Wu).

\section{Footnote}

Reporting Checklist: The authors have completed the STROBE reporting checklist. Available at http://dx.doi. org/10.21037/tau-21-142

Peer Review File: Available at http://dx.doi.org/10.21037/ tau-21-142

Conflicts of Interest: All authors have completed the ICMJE uniform disclosure form (available at http://dx.doi. org/10.21037/tau-21-142). YX serves as an unpaid editorial board member of Translational Andrology and Urology. The other authors have no conflicts of interest to declare.

Ethical Statement: The authors are accountable for all aspects of the work in ensuring that questions related to the accuracy or integrity of any part of the work are appropriately investigated and resolved. The study was conducted in accordance with the Declaration of Helsinki (as revised in 2013). The study was approved by Institutional Review Board (IRB) of the Tianjin First Center Hospital (registration ID2018NO22KY) and individual consent for this retrospective analysis was waived.

Open Access Statement: This is an Open Access article distributed in accordance with the Creative Commons Attribution-NonCommercial-NoDerivs 4.0 International License (CC BY-NC-ND 4.0), which permits the noncommercial replication and distribution of the article with the strict proviso that no changes or edits are made and the original work is properly cited (including links to both the formal publication through the relevant DOI and the license). See: https://creativecommons.org/licenses/by-nc-nd/4.0/.

\section{References}

1. Malde S, Nambiar AK, Umbach R, et al. Systematic Review of the Performance of Noninvasive Tests in Diagnosing Bladder Outlet Obstruction in Men with Lower Urinary Tract Symptoms. Eur Urol 2017;71:391-402.

2. Ko YH, Kim TH, Song PH, et al. Structural Variations of the Prostatic Urethra Within the Prostate Predict the Severities 
of Obstructive Symptoms: A Prospective Multicenter Observational Study. Urology 2017;104:160-5.

3. Ko YH, Song PH. Structural Variation of Prostate Urethra Reflected by the Ratio Between Prostate Volume and Prostatic Urethral Length is Associated with the Degrees of Lower Urinary Tract Symptoms. Low Urin Tract Symptoms 2016;8:113-9.

4. Minagawa T, Daimon H, Ogawa N, et al. Morphological and clinical evaluation of prostatic urethra using modified sonourethrography with retrograde jelly injection. Low Urin Tract Symptoms 2019;11:O4-10.

5. Agrawal V, Khullar R, Jha AK. Assessment of posterior urethra in benign prostatic hyperplasia and after its surgery. Urol Ann 2020;12:63-8.

6. David RA, Badmus TA, Salako AA, et al. Diagnostic performance of transrectal ultrasound for prostate volume estimation in men with benign prostate hyperplasia. Int J Clin Pract 2020;74:e13615.

7. Chen L, Feng C, Lv XG, et al. Three-Dimensional Computerized Model Based on the Sonourethrogram: A Novel Technique to Evaluate Anterior Urethral Stricture. J Urol 2018;199:568-75.

8. Ni J, Bongers A, Chamoli U, et al. In Vivo 3D MRI Measurement of Tumour Volume in an Orthotopic Mouse Model of Prostate Cancer. Cancer Control 2019;26:1073274819846590.

9. Anzia LE, Johnson CJ, Mao L, et al. Comprehensive noninvasive analysis of lower urinary tract anatomy using MRI. Abdom Radiol (NY) 2021;46:1670-6.

10. Martins T, Mussi TC. Prostate volume measurement by multiparametric magnetic resonance and transrectal ultrasound: comparison with surgical specimen weight. Einstein 2020;18:eAO4662.

11. Bezinque A, Moriarity A, Farrell C, et al. Determination of Prostate Volume: A Comparison of Contemporary Methods. Acad Radiol 2018;25:1582-7.

12. Malalasekera AP, Sivasuganthan K, Sarangan S, et al. Morphological variations of the human ejaculatory ducts in relation to the prostatic urethra. Clin Anat 2018;31:456-61.

13. Stanzione A, Ponsiglione A, Di Fiore GA, et al. Prostate Volume Estimation on MRI: Accuracy and Effects of Ellipsoid and Bullet-Shaped Measurements on PSA Density. Acad Radiol 2020;S1076-6332(20)30290-7.

14. Aprikian S, Luz M, Brimo F, et al. Improving ultrasoundbased prostate volume estimation. BMC Urol 2019;19:68.

15. El-Adawy MS, Abdelaziz AY, Salem A, et al. Relation of baseline prostate volume to improvement of lower urinary tract symptoms due to tamsulosin monotherapy in benign prostatic hyperplasia: An exploratory, multicenter, prospective study. Urol Ann 2020;12:271-5.

16. Wang B, Zhang S, Sun C, et al. Comparison between a transurethral prostate split and transurethral prostate resection for benign prostatic hyperplasia treatment in a small prostate volume: a prospective controlled study. Ann Transl Med 2020;8:1016.

17. Stone NN, Crawford ED, Skouteris VM, et al. The Ratio of the Number of Biopsy Specimens to Prostate Volume (Biopsy Density) Greater Than 1.5 Improves the Prostate Cancer Detection Rate in Men Undergoing Transperineal Biopsy of the Prostate. J Urol 2019;202:264-71.

18. Gunseren KO, Akdemir S, Çiçek MC, et al. Holmium Laser Enucleation, Laparoscopic Simple Prostatectomy, or Open Prostatectomy: The Role of the Prostate Volume in terms of Operation Time. Urol Int 2021;105:285-90.

19. Topazio L, Perugia C, De Nunzio C, et al. Intravescical prostatic protrusion is a predictor of alpha blockers response: results from an observational study. BMC Urol 2018;18:6.

20. Tian Y, Liu HM, Yang B, et al. Prostatic anatomical parameters correlate with clinical characteristics suggestive of benign prostatic hyperplasia. Asian J Androl 2021;23:64-8.

21. Kwon JK, Han JH, Choi HC, et al. Clinical significance of peripheral zone thickness in men with lower urinary tract symptoms/benign prostatic hyperplasia. BJU Int 2016;117:316-22.

22. Matsugasumi T, Fujihara A, Ushijima S, et al. Morphometric analysis of prostate zonal anatomy using magnetic resonance imaging: impact on age-related changes in patients in Japan and the USA. BJU Int 2017;120:497-504.

23. Williams AM, Simon I, Landis PK, et al. Prostatic growth rate determined from MRI data: age-related longitudinal changes. J Androl 1999;20:474-80.

24. Shi YF, Yu DJ. TRAF6 regulates proliferation of stromal cells in the transition and peripheral zones of benign prostatic hyperplasia via Akt/mTOR signaling. Prostate 2018;78:193-201.

25. Watanabe H. New concept of BPH: PCAR theory. Prostate 1998;37:116-25.

26. Shim M, Bang WJ, Oh CY. Correlation between prostatic urethral angulation and symptomatic improvement after surgery in patients with lower urinary tract symptoms according to prostate size. World J Urol 2020;38:1997-2003.

27. Chen H, Zhang CA, Gill HS. Anatomical study of the prostatic urethra using vinyl polysiloxane casts. Prostate 
2020;80:241-6.

28. El-Tatawy H, Gameel T, El-enen MA, et al. Clinical significance of prostatic-urethral angulation on the treatment outcome of patients with symptomatic benign prostatic hyperplasia treated with tamsulosin hydrochloride. Arch Ital Urol Androl 2015;87:238-42.
29. Guneyli S, Ward E, Peng Y, et al. MRI evaluation of benign prostatic hyperplasia: Correlation with international prostate symptom score. J Magn Reson Imaging 2017;45:917-25.

30. Fry CH, McCloskey KD. Spontaneous Activity and the Urinary Bladder. Adv Exp Med Biol 2019;1124:121-47.

Cite this article as: Feng $\mathrm{Y}, \mathrm{Wu} \mathrm{J}$, Zhu H, Wang Q, Li T, Xu Y, Zhang P, Zhai L. Three-dimensional measurement and analysis of benign prostatic hyperplasia. Transl Androl Urol 2021;10(6):2384-2396. doi: 10.21037/tau-21-142 